\title{
Dynamic Time Warping for Heartbeat Detection in Ballistocardiography
}

\author{
Guillaume Cathelain ${ }^{1}$, Bertrand Rivet ${ }^{2}$, Sophie Achard ${ }^{2}$, Jean Bergounioux ${ }^{3}$, François Jouen ${ }^{1}$ \\ ${ }^{1}$ CHArt Laboratory, Ecole Pratique des Hautes Etudes, PSL University, Paris, France \\ ${ }^{2}$ Université Grenoble Alpes, CNRS, Grenoble INP, GIPSA-lab, Grenoble, France \\ ${ }^{3}$ Pediatric Intensive Care Unit, Assistance Publique des Hôpitaux de Paris, Garches, France
}

\begin{abstract}
Monitoring vital signs of neonates can be harmful and lead to developmental troubles. Ballistocardiography, a contactless heart rate monitoring method, has the potential to reduce this monitoring pain. But signal processing is uneasy due to noise, inherent physiological variability and artifacts (e.g. respiratory amplitude modulation and body position shifts). We propose a new detection of heartbeats using a dynamic time warping (DTW) template matching method. A heartbeat template is automatically trained and the most similar potential heartbeats in the signal are classified as heartbeats. Data normalizing and DTW steps overcome amplitude and time variability issues. The DTW template matching algorithm has been tested on a 20 to 50 minutes-long BCG records of ten healthy adults in noisy conditions. It is suitable for medical, real-time and low-cost applications.
\end{abstract}

\section{Introduction}

The physiological state (cardiac and respiratory activities) of children in hospital environment is usually evaluated by electrocardiography (ECG) and photoplethysmography (PPG). The former uses electrodes on the thorax and on the limbs extremities; the latter uses pulse oximetry probes that tweak either a finger or a toe. Monitoring children is difficult because of their high mobility that leads to repetitive artifacts and false alarms. Moreover, electrodes peel off the epidermis and pulse oximetry probes can regularly detach from the finger or toe. Several factors are involved: the child twitches and pulls the probes, the electrode adhesive is worn or the nursing staff could not properly set up the electrodes. Children pain, which may be monitored by heart rate variability (HRV) [1] or infrared thermography [2], affects the neuro-motor and cognitive development especially for preterm neonates [3]. Finally, this equipment is expensive to install and maintain; given the elements described above, it must be changed several times during hospitalization. Consequently, a nonintrusive apparatus is necessary to detect children heartbeats in a harmless way.

Ballistocardiography is a non-intrusive monitoring method for cardiac activity. It was invented at the end of the 19th century but supplanted by the first electrocardiographs, improving precision and robustness at that time. Today, with new sensor technologies and digital signal processing, this technology is gaining renewed interest. Ballistocardiography's principle relies on measuring ballistic forces [4]: during ventricular systole, blood is ejected from the left ventricle through the aortic arch, generating a pulsed cardiac ballistic force. This mechanical phenomenon allows contactless measurements of a pressure variation (e.g strain gauge) or a deformation (e.g accelerometers) of the patient bed or mattress. A ballistocardiogram (BCG) is a record of this mechanical phenomenon characterized by $\mathrm{H}$ to $\mathrm{N}$ peaks; however a BCG signal is generally affected by noise such as respiratory, movement artifacts and hardware limitation. Few studies [5] focus on pediatric ballistocardiography, where BCG signals are noisier. Compared to adults, the BCG signal amplitude of a $3 \mathrm{~kg}$ infant is about 30 times lower due to low weight and low cardiac contractile force [6].

Specific digital signal processing algorithms have been developed for detecting heartbeats, beat-to-beat heart rate and heart rate variability (HRV) in BCG signals using time domain or time-frequency domain methods [7]. In template matching methods, heartbeats' shape is manually $[8,9]$ or automatically [10] modeled and crosscorrelated to the signal. However the cross-correlation function may be difficult to threshold because of amplitude and time variability of the IJK complex.

In this context, we present a new method to detect heartbeats based on a template matching algorithm improved by DTW and heartbeat normalization. The resulting detection is robust to noise and heartbeats variability. The remaining of this paper is organized as follows: Section 2 presents the materials and Section 3 details the proposed method before results in Section 4. Finally, Section 5 concludes this study. 


\section{Materials}

A MM0 3000 medical bed has been equipped with a Murata SCA11H BCG sensor. The sensor is placed on top of a MMO Aerospacer overlay in a head-to-foot direction as shown in Figure 1. This wireless sensor is based on a SCA61T accelerometer with a $150 \mu \mathrm{m} / \mathrm{s}^{2} / \sqrt{ } \mathrm{Hz}$ low noise density. The analogue output is AC coupled, anti-aliasing filtered and amplified before digitization at 1 $\mathrm{kHz}$.

The digital signal is filtered with third order Butterworth filters, specifically a $25 \mathrm{~Hz}$ low-pass filter and a $2 \mathrm{~Hz}$ high-pass filter. They are applied forward and backward in order to prevent phase distortion. Lastly, the signal is decimated to $200 \mathrm{~Hz}$ sampling frequency.

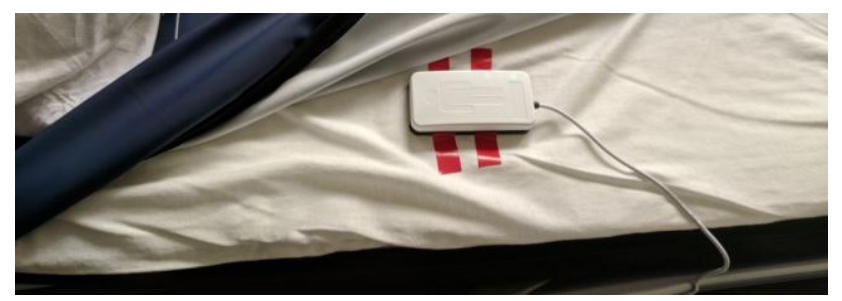

Figure 1. Position of the BCG sensor.

Ten healthy adult volunteers were monitored for 20 to 50 minutes-long naps during lunch break in Ecole Pratique des Hautes Etudes. This process has been validated by an ethical commission in conformity with the European data legislation. Volunteers were asked to lie down on the bed and rest in supine position.

\section{Methods}

The ultimate goal of the methods is to accurately detect heartbeats in BCG, for heart rate and HRV analyses.

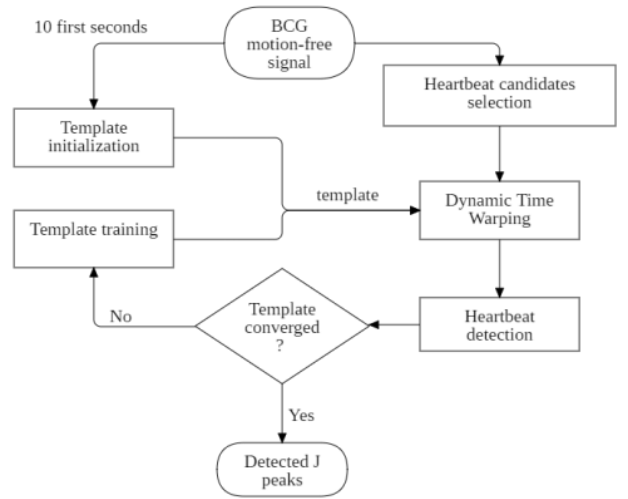

Figure 2. Flowchart of the methods.

Prior to the detection algorithm, motion-free BCG signals were manually isolated. In this paper we focus on these signals. Figure 2 illustrates the methods. Final steps of template training and Signal-to-Noise (SNR) ratio estimation are detailed in Sections 3.5 and 3.6.

\subsection{Template initialization}

In template matching methods, a template helps recognize similar patterns in the signal; it is often defined manually. In our case, a heartbeat template is automatically initialized for each participant in the following manner. An envelope detection algorithm based on a Hilbert transform is applied on the ten first motionfree seconds of the BCG signal. Local minima, spaced by a minimum delay $\Delta \mathrm{t}$ depending on a hr estimate of the heart rate, are detected. The HRestimate equals 60 times the fundamental frequency of the BCG envelope's Fast Fourier Transform.

Segments starting from one of these local minima to another one are very likely to include one heartbeat each and are thus called inter-beat intervals IBI. Global minima of IBIs are probably $\mathrm{J}$ peaks, as illustrated in Figure 2.

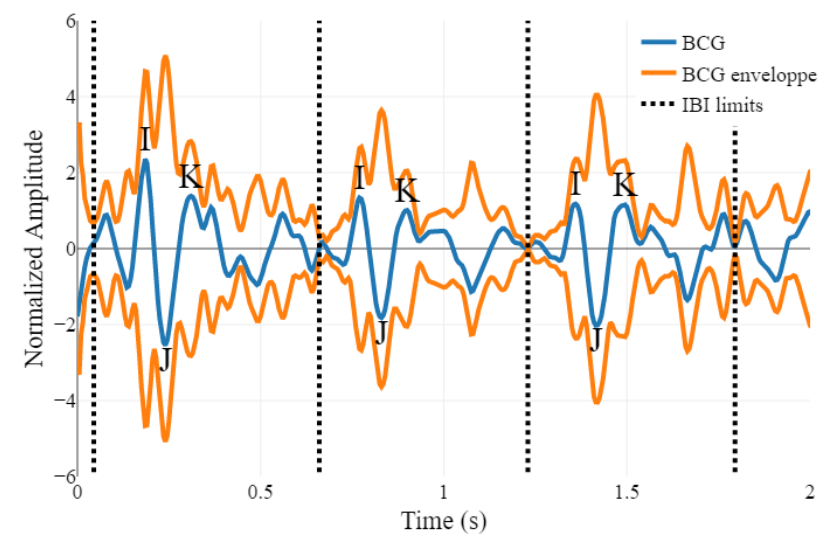

Figure 3. BCG envelope detection and segmentation.

In Figure 3, IBIs are $\mathbf{J}$ peaks synchronized, z-score normalized and averaged altogether. Normalizing IBIs is necessary to preserve the shape of heartbeats and to overcome amplitude variability issues. The initial heartbeat template is parametrized by its length $\mathrm{L}$ and the $\mathrm{J}$ peak index $\mathrm{i}_{\mathrm{J}}$.

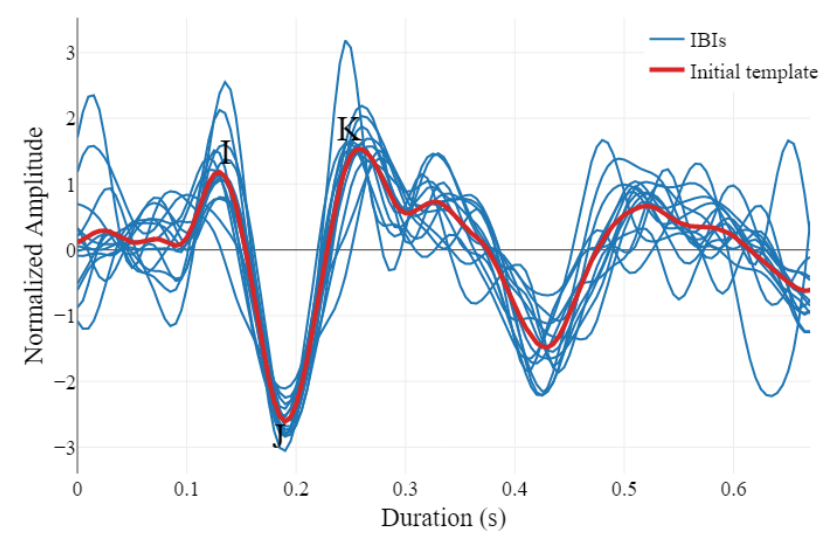

Figure 4. Initialization of the template. 


\subsection{Heartbeat candidates selection}

In order to recognize patterns similar to the initialized template, the BCG signal is sliced into L-long IBIs called heartbeat candidates. Local minima of the BCG signals are detected as potential $\mathrm{J}$ peaks of heartbeat candidates. Heartbeat candidates are IBIs shortened to L with J peak at index $\mathrm{i}_{\mathrm{J}}$. There are potentially about 25 heartbeat candidates per second because low-pass cut off frequency is $25 \mathrm{~Hz}$; features like minimum distance between peaks or minimum height of peaks can help reduce the number of candidates.

\subsection{Dynamic Time Warping}

Heartbeat candidates must be compared with the template in such a way that time variability issues are overcome. Dynamic Time Warping (DTW) is a method for measuring similarity between two time series which are globally or locally delayed. By aligning the sequences in time, DTW is independent of the non-linear time variability of the sequences. It is widely used for pattern recognition in time series. It has been used in BCG for evaluating the potential changes in the signal induced by different respiratory patterns and posture [11].

Optimization, in terms of normalization, endpoints weighting and warping constraint has been performed [12]. Firstly, the initial template and heartbeat candidates are z-score normalized and multiplied by a tapered cosine window in order to smooth endpoints and force a zero value. Secondly, the width of the warping path is constrained to 5 samples by a Sakoe-Chiba band, which corresponds to $25 \mathrm{~ms}$ local delays. In fact, as the maximum frequency of the signal is limited to $25 \mathrm{~Hz}$, adjacent local minima are minimally spaced by $40 \mathrm{~ms}$. If time warping were longer than this delay, heartbeat candidates might be confounded.

\subsection{Heartbeat detection}

Heartbeat candidates are sorted in a buffer by their DTW distance with the template. Starting from the smallest DTW distance, heartbeats candidates are discarded from the buffer as soon as they are closer in time than $\Delta \mathrm{t}$ to a heartbeat candidate with smaller DTW distance.

Beat-to-beat delays, heart rate series and HRV are computed from $\mathrm{J}$ peak position of the remaining heartbeat candidates.

\subsection{Template training}

The initial template is an average of IBIs synchronized with their probable $\mathbf{J}$ peaks for the ten first seconds. Its shape may differ from actual heartbeats, consequently the template must be refined. In the template training step, IBIs are updated with the new detected $J$ peaks and the trained template is the average of IBIs synchronized with these $\mathrm{J}$ peaks. The previous steps of optimization, DTW execution and heartbeat detection are then iterated to make sure that no false positive or false negative occurred when the template differed from actual heartbeats. Iterations are executed until the template converges (two iterations are generally enough for a ten-second-long BCG signal). An example is given in Figure 4.

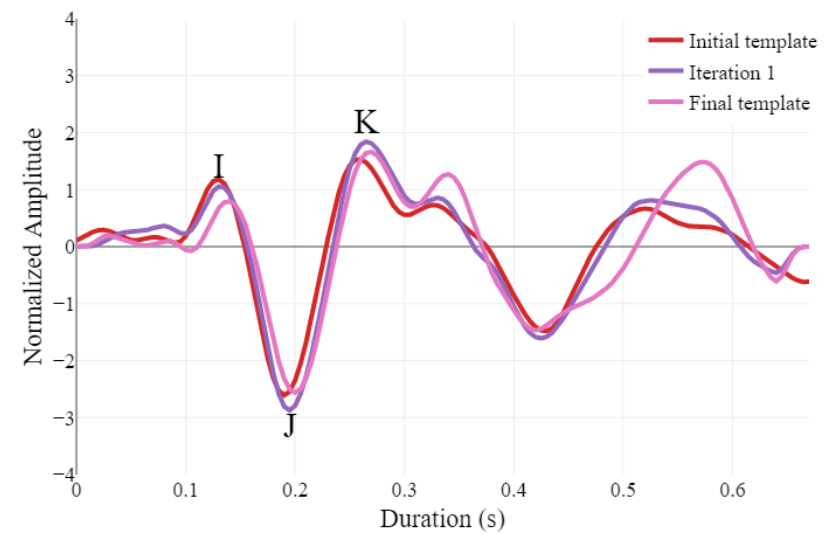

Figure 5. Refinement of the template.

\subsection{Signal-to-noise ratio estimation}

Noisy conditions must be quantified to check the algorithm performance. SNR estimation was estimated for each motion-free BCG signal as the average sample correlation coefficient between pairs of sequential heartbeats [13].

\section{Results}

The DTW template matching algorithm presented in this paper has been tested on the BCG database described in 2.2. A medical expert manually checked every heartbeat using a dedicated data visualization software. A detected heartbeat is classified as a true positive only if its $\mathrm{J}$ peak is at the same location of the $\mathrm{J}$ peak of a real heartbeat. Over all records ( $\mathrm{M}=14139$ real heartbeats), false positives (FP), true positives (TP) and false negatives (FN) are reported in Table 1. Specificity was not computed because true negatives are ill-defined.

Table 1. Truth table for detection of heartbeats.

\begin{tabular}{|l|l|l|}
\hline $\mathrm{M}=14139$ & Heartbeat & No heartbeat \\
\hline Detected & $\mathrm{TP}=13523$ & $\mathrm{FP}=433$ \\
\hline Undetected & $\mathrm{FN}=616$ & \\
\hline
\end{tabular}

Overall sensitivity and positive predictivity are $95.6 \%$ and $96.8 \%$ respectively, with records' SNR ranging from 
2.0 to 14.6 . If we separate records whose SNR are lower than 3 (four records) from those higher than 3 (six records), sensitivity and positive predictivity will be $85.3 \%$ and $88.5 \%$ in the noisiest case; $98.7 \%$ and $99.2 \%$ in the second case.

\section{Discussion}

We presented a template matching method for automatically detecting and determining the shape of heartbeats in BCG signals, which solves amplitude and time variability issues by dynamic time warping and normalization. This algorithm was successfully tested on a database with varying conditions of noise.

Synchronized gold standard physiological measurements such as ECG were missing, so detected heartbeats were checked manually. This process is tedious especially in noisy conditions where it is difficult to manually label heartbeats. Moreover, the heartbeat detection step of the algorithm forces a minimum delay between two consecutive heartbeats based on an estimation of the average heart rate. This strong assumption might be locally refined using Short-Term Fourier Transform depending on the length of the signal. In addition as heartbeats are selected by their ascending DTW distance with the template, one badly labeled heartbeat often leads to multiple false positives in its neighborhood; this issue could be solved by adding features for the heartbeat detection step.

The adult BCG database, realized in noisy ecological conditions, served as a proof of concept for this algorithm, which will be further tested on infants and neonates BCG databases during experimentations in Raymond Poincaré Hospital - APHP in Garches, France. The presented algorithm enables the possibility to detect heartbeats in BCG signals recorded by usual industrial MEMS sensors that are noisier but far less expensive.

\section{Acknowledgments}

The database recording would not have been possible without the support of Ecole Pratique des Hautes Etudes and its volunteers. Medical beds and overlays were lent by MMO, France. The whole study has been financed by Institut Carnot Cognition and PSL University.

\section{References}

[1] B. M. Appelhans, and L. J. Luecken, "Heart rate variability and pain: associations of two interrelated homeostatic processes”, Biological Psychology, vol. 77, no. 2, pp 174182, Feb. 2008.

[2] S. Brummelte, R. E. Grunau, V. Chau, K. J. Poskitt, R. Brant, J. Vinall, A. Gover, A. R. Synnes, and S. P. Miller, "Procedural pain and brain development in premature newborns," Annals of Neurology, vol. 71, no. 3, pp 385-
396, Feb. 2012.

[3] A. Alalwani, Y. Chahir, B. Guillois, M. Molina, and F. Jouen, "Neonatal pain recognition using LBP descriptor and wavelet thresholding technique", IEEE International Conference on Multimedia Computing and Systems, Apr. 2014.

[4] C-S. Kim, S. L. Ober, M. S. McMurtry, B. A. Finegan, O. T. Inan,and R. M. Hahn,"Ballistocardiogram: mechanism and potential for unobtrusive cardiovascular health monitoring", Nature Scientific Reports, vol. 6, Aug. 2016, Art. No. 31297.

[5] W. K. Lee, H. Yoon, D. W. Jung, S. H. Hwang, and K. S. Park, "Ballistocardiogram of baby during sleep", 37th Annual International Conference of the IEEE Engineering in Medicine and Biology Society, 2015.

[6] M. Erkinjuntti, K. Vaahtoranta, J. Alihanka, and P. Kero, "Use of the SCSB method for monitoring of respiration, body movements and ballistocardiogram in infants,"Early Human Development, vol. 9, pp 119-126, Feb. 1984.

[7] O. T. Inan, P-F. Migeotte, K-S. Park, M. Etemadi, K.Tavakolian, R.Casanella, J.Zanetti, J. Tank, I.Funtova, G. K. Prisk, and M. DiRienzo, "Ballistocardiography and seismocardiography: a review of recent advances", IEEE Journal of Biomedical and Health Informatics, vol. 19, no. 4, pp. 1414-1427, Jul. 2015.

[8] B. Hans Jansen, and K. Shankar, "Monitoring of the ballistocardiogram and with the static and charge sensitive and bed", 748 IEEE Transactions On Biomedical Engineering,vol. 38, no. 8., Aug. 1991.

[9] J. H. Shin, B. H. Choi, Y. G. Lim, D. U. Jeong, and K. S. Park,"Automatic ballistocardiogram (BCG) beat detection using a template and matching approach", 30th Annual International IEEE EMBS Conference Vancouver, Aug. 2008.

[10] J. Paalasmaa, H. Toivonen, and M. Partinen "Adaptive heartbeat modeling for beat-to-beat heart rate measurement in ballistocardiograms", IEEE journal of biomedical and health informatics, vol. 19, no. 6, pp.1945-1952, 2015.

[11] A. Martín-Yebra, F. Landreani, C. Casellato, E. Pavan, P-F. Migeotte, C. Frigo, J. P. Martínez, and E. G. Caiani, "Evaluation of respiratory- and postural-induced changes on the ballistocardiogram signal by time warping averaging", Physiological Measurement, vol. 38, pp. 14261440, Jun. 2017.

[12] A. Mueen, E. Keogh, "Extracting optimal performance from dynamic time warping", Proceedings of the 22nd ACM SIGKDD International Conference on Knowledge Discovery and Data Mining, 2016.

[13] C. McCall, Z. Stuart, R. M. Wiard, O. T. Inan, L. Giovangrandi, C. M. Cuttino, and G. T. A. Kovacs, "Standing ballistocardiography measurements in microgravity", 36th Annual International Conference of the IEEE Engineering in Medicine and Biology Society, 2014.

Address for correspondence:

Guillaume Cathelain, PhD student

Ecole Pratique des Hautes Etudes

4-14 rue Ferrus, 75014 Paris, France

guillaume.cathelain@ephe.psl.eu 